http://www.jfas.info

\title{
METHOD TO CONTROL AND CORRECT TELEMTRY WELL INFORMATION IN THE BASIS OF RESIDUE NUMBER SYSTEM
}

\author{
K. T. Tyncherov*, V. Sh. Mukhametshin, L. B. Khuzina \\ Federal State Budgetary Educational Institution of Higher Education Ufa State Petroleum \\ Technological University (USPTU) in Oktyabrskii
}

Published online: 08 August 2017

\begin{abstract}
The paper considers the issue of increasing the reliability of the borehole telemetry information. The issue is suggested to be solved by applying the code of nonpositional residue number system instead of a traditionally used positional number notation to code the information. The residue number system is shown to have the best error-correcting capacities and is able both to detect the errors and correct them.
\end{abstract}

Key words: oil wells; telemetry information, coding, residue number system, error-correcting capacities, error detection, error correction.

\section{Brief outline:}

1) Reception and processing of telemetry information from the oil wells equipped with bottom-hole pumps are connected with low reliability of the wireless transmitted signals;

2) Actual information coding methods based on a traditional positional number notation can not completely correct the errors occurred during digital processing of the signals;

3) The nonpositional residue number system has better potential both to detect and to correct the errors.

4) The application of the residue number system increases the reliability of the telemetry information transmission from the oil wells equipped with the bottom-hole pumps.

The transmission of the telemetry borehole information to the surface is one of the most crucial questions in carrying out the hydrodynamic analysis of the oil wells equipped with the bottom-hole pumps.

Author Correspondence, e-mail: author@gmail.com

doi: http://dx.doi.org/10.4314/jfas.v9i2s.848 
Telemetry information represents the data from the sensors later transformed into a digital form for such parameters as temperature, pressure, consumption rate, water content in a mixture, etc. The wells with bottom-hole pumps are designed to use isolated parker-type devices with no possibility to put the information cables. Therefore, to transmit the information from the borehole telemetry tools, the wireless channels with self-generated power supply of electric circuitry are normally used.Broadband noise-like signals based on cycling Bose-Chaudhuri-Hocquenghem codes (BCH-codes) and low frequency repetitive transmission are used to improve the reliability of the transmitted information to the surface. However, this is typically not adequate. Due to the interference of various nature, the received signals are often increasingly distorted, and the obtained false information can negatively affect the results of the well's parameters analysis.

One of the reasons causing low reliability of the transmitted information is the usage of standard positional number notation with poor capacity to self-correction for the signal coding. Hence, the challenge to find the number notation system with the ability to self-detect and self-correct the errors occurred during the digital processing of the signals is very relevant [2].

One of the possible solutions for this task is to study the possibilities for telemetry information coding based on anon positional number notation system with potential correcting properties.

The code of residue number system is the most attractive one for the existing correcting codes.

The residue number system (RNS) is based on the Chinese theory about the residue offered by Sun Tzu, a Chinese mathematician, in about III-V centuries AD. In this system the operands are in the form of residues or remainders $\alpha_{i}, i=\overline{1, n}$ from the division of the operand $A$ by a set of natural relative primes $m_{i}$ called the bases or modules of RNS, in other words $A=\left(\propto_{1}, \propto_{2}, \ldots, \propto_{n}\right)$, where the biggest common divisor is $\left(m_{j}, m_{k}\right)=1, j \neq k$.

Coding in RNS means that if a multitude $L$ contains a set of natural numbers $A$, for example, $A_{1}, A_{2} \in L$, then any number $A_{3}$ between $A_{1}$ and $A_{2}$ also belongs to the multitude $L$, that is, $A_{3} \in L$. Generally, any number $A_{k} \in L$ may be presented in RNS with the base $M=$ $\left[m_{1}, m_{2}, \ldots m_{n}\right]$, if $L \leq M$, where $M$ is the lowest common divisible for the natural numbers $m_{1}, m_{2}, \ldots m_{n}\left(P_{n}=\prod_{i=1}^{n} m_{i}\right)$.

The specified ratios are basic for generating the codes capable of correcting the errors occurred during the digital processing of the information in well telemetry systems. 
Correcting codes in RNS are divided into three classes by the ratio between the values $L, M u P_{n}$ :

- nonlinear codes $\left(L=M=P_{n}\right)$;

- half-linear codes $\left(L<M<P_{n}\right)$;

- linear codes $\left(L=M<P_{n}\right)[4]$.

Nonlinear code or $R$ is the code with vector $\Gamma$ corresponding to the numbers presented in RNS with mutually simple (with no common divisor) bases $m_{1}, m_{2}, \ldots m_{n}$.

These codes may have the minimal code distance $d_{\min }$ depending on the degree of redundancy $R=P_{n} / \mathrm{L}$, with any specified RNS the value $R$ unequivocally determines the correcting capacities $R$ of the code.

Indeed, $R$ code has its minimal distance, when the degree of redundancy is no less than any products of any $d_{\text {min }}-1$ bases determined by RNS, or $R \geq \prod_{i=1}^{d-1} m_{q_{i}}$, where $q_{i}=1,2, \ldots n$.

RNS bases $m_{1}, m_{2}, \ldots m_{n}$ are informational, while the bases $m_{n+1}, m_{n+2}, \ldots m_{n+k}$ are controlling.

If RNS is arranged, then $d_{\text {min }}=k+1$, and if the system expands by adding $k$ bases, and every base is bigger than any informational base, then the minimal code distance automatically extends by the value $k . d_{\min }$ can be increased with the decrease in the number of the informational bases, that is moving to the less accurate calculations. Thus, there is an inverse proportion between the correcting capacities $R$ of the codes and the calculation accuracy. It means that one and the same processor can digitally process the telemetry signals with high accuracy, but with low reliability or with less accuracy, but with more reliability and at a higher speed.

To detect the errors RNS range $M=\left[m_{1}, m_{2}, \ldots m_{n}\right]$ extends due to the introduction of the base mutually simple with other modules $m_{n+1}>m_{n}$. Then the RNS range is defined as $M=M \cdot m_{n+1}$.

The identification of the wrong number is based on the fact that RNS is arranged; that is, if the system $A=\left(\propto_{1}, \propto_{2}, \ldots, \propto_{i}, \ldots, \propto_{n+1}\right)$ is adequately arranged, then the number $\tilde{A}=$ $\left(\propto_{1}, \propto_{2}, \ldots, \widetilde{\propto}_{i}=\propto_{i}, \ldots, \propto_{n+1}\right)$ is wrong. Hence, to detect the distorted number $A=$ $\left(\propto_{1}, \propto_{2}, \ldots, \propto_{n}, \ldots, \propto_{n+1}\right)$ it is necessary to compare it with the operating range $M$. If $A \geq M$, then the number is distorted, and if $A<M$, then there is no error or the error is of complex nature. 
To correct the error it is necessary to introduce one more control base $m_{n+2}>m_{n+1}$. Surely, the whole range of RNS changes: $M_{2}=M_{1} \cdot m_{n+2}$. Then the number projections $\tilde{A}=$ $\left(\propto_{1}, \propto_{2}, \ldots, \propto_{n}, \propto_{n+1}, \propto_{n+2}\right)$ are calculated at all RNS bases:

$\tilde{A}_{1}=\left(\propto_{2}, \propto_{3}, \ldots, \propto_{n}, \propto_{n+1}, \propto_{n+2}\right)$;

$\tilde{A}_{2}=\left(\propto_{1}, \propto_{3}, \ldots, \propto_{n}, \propto_{n+1}, \propto_{n+2}\right)$;

$\cdots$

$\tilde{A}_{n+1}=\left(\propto_{1}, \propto_{2}, \ldots, \propto_{n}, \propto_{n+2}\right)$;

$\tilde{A}_{n+2}=\left(\propto_{1}, \propto_{2}, \ldots, \propto_{n}, \propto_{n+1}\right)$.

The obtained projections $\tilde{A}_{i}=(\overline{1, n+2})$ are compared with the operating range $M$.

With the number projection for which $\tilde{A}_{i}<M$, the error residue is corrected:

$\alpha_{i}=\tilde{\alpha}_{i}+\left[\frac{m_{i}\left(1+j m_{n+1}\right)}{m_{n+1} \overline{m_{l}}}-\frac{\tilde{A}}{B_{i}}\right]$,

where $B_{i}=\overline{m_{l}} \mathrm{M} / m_{i}$ is the orthogonal RNS base, $j=0,1,2, \ldots, n$; $\overline{m_{l}}$ is the weight of the orthogonal base.

In addition to the above, it should also be noted that the studied $R$ code has the arithmetic property. This property is that the bases are included into the common bases system of RNS, where the codes with the numbers at all basic and check bits participate in any operation of telemetry information processing. Besides, the basic and additional numbers are processed in the same way. It means that in RNS the information is processed at stages with no control from any separate code. The length of the stage can be determined in any particular case by the complete processing cycle for all telemetry information or in accordance with the probability for single error occurrence. The final result of any calculating stage can be controlled, and its adequacy can evidence the adequacy for all operations of this stage.

Generally, the introduction of at least one control base helps to detect not only one single error, but the majority of the double errors [3-5].

This point is significant to choose the coding method for well telemetry information, usage of nonpositional residue number system. The coding implementation requires the introduction of digital processing for direct and inverse converter signals into the scheme, with the converters not significantly affecting the speed characteristics of a processor. The detection and correction of the occurred errors will increase the reliability of telemetry information reception, thus hydrodynamic study of an oil well equipped with bottom-hole pumps will be carried out with the appropriate quality. 


\section{REFERENCES}

1 Shakirov A.A., Development of geophysical complex of collectors hydrodynamic studies [Text] / A.A. Shakirov, K.T. Tyncherov, M.V. Goryunova //Oil and gas business: electronic

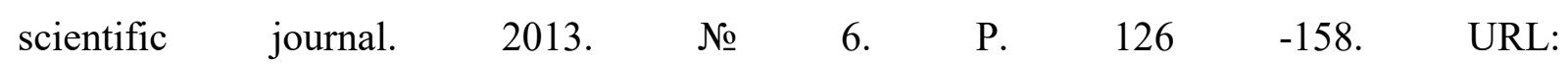
http://www.ogbus.ru/authors/ShakirovAA/ShakirovAA_2.pdf

2 Chervyakov, N.I., Veligosha, A.V., Tyncherov, K.T., Velikikh, S.A. Use of modular coding for high-speed digital filter design //Cybernetics and Systems Analysis, 1988,- № 2, - PP. 110.

3 Chervyakov, N.I., Tyncherov, K.T., Veligosha, A.V.High-speed digital signal processing with using non-positional arithmetic //Radiotekhnika, 1997, -№ 10. - PP. 23-28.

4 Tyncherov K.T. On rationale for the neural network calculations on the basis of nonpositional number notation in remainders [Text] / K.T. Tyncherov // Automation, telemachanization and communication in oil industry. - M.: OAO "VNIIOENG", 2011. - №2. - p.34-37.

5 Tyncherov K.T. Evaluation of modular neuroprocessor ASUTP reliability [Text] / K.T. Tyncherov // Bulletin of Moscow Aviation Institute. M.: MAI, 2011, vol.18, №3, p.219-221.

\section{How to cite this article:}

Tyncherov K T,. Mukhametshin V Sh, Khuzina L B. Method to control and correct telemtry well information in the basis of residue number system. J. Fundam. Appl. Sci., 2017, 9(2S), 1370-1374. 